ISSN: 2683-2321

\title{
Ingeniería de la formación: emprender un dispositivo de capacitación acorde a las necesidades actuales de los profesores de francés en México \\ Training engineering: undertaking a training plan according to the current needs of French teachers in Mexico
}

${ }^{a}$ Edward Amador-Pliego, ${ }^{b}$ Delphine-Pluvinet

\section{Resumen:}

En los últimos años, han surgido nuevas situaciones sociales y académicas en México, permitiendo una movilidad más competitiva para los estudiantes de lenguas, en particular del francés, promoviendo intercambios y objetivos de aprendizaje diversos.

Ante estas nuevas situaciones, muchos docentes están haciendo enormes esfuerzos para integrar nuevas estrategias en su planeaciones y dinámicas de clase, permitiendo que los alumnos se adapten mejor a estos nuevos contextos sin necesariamente estar preparados institucionalmente.

La presente investigación comparte los primeros hallazgos iniciados a finales del 2019 como parte de un Encuentro de Licenciaturas de Francés Lengua Extranjera (FLE) en México. A raíz de ese encuentro nacional surgieron muchas preguntas sobre la formación y el perfil propio de los docentes de FLE en México, las cuales definieron nuestra investigación.

El principal objetivo del enfoque es analizar las necesidades, diseñar acciones y esquemas de formación, e implementarlos y evaluarlos siguiendo un enfoque razonado y estructurado, contando con la participación de los propios docentes que se involucraron en nuestro trabajo.

Para comenzar, se trató de establecer el perfil actual del docente de FLE en México. La muestra para construir dicho perfil fue constituida por 100 profesores de ocho universidades públicas de diversos estados del país. Se tomaron en cuenta las observaciones de estos docentes respecto al perfil "idóneo" de un docente de FLE en México y se revisó su propia trayectoria de formación como docente de FLE. Además, se consideraron las diversas concepciones que los directores, coordinadores y responsables de las facultades de idiomas y centros de lenguas tienen del perfil docente necesario para que sus instituciones contraten a su personal para impartir clases ya sea en centros de autoaprendizaje o laboratorios de FLE.

Esta investigación tiene también como objetivo proponer una nueva representación del perfil de los docentes de FLE en México, que sirva para comprender las diferentes influencias que determinan las prácticas pedagógicas de los docentes. Partiendo de varios modelos existentes, pero principalmente de los principios y herramientas de la Ingeniería de la Formación. Parmentier (2012) afirma que "se debe comprender los métodos y prácticas de análisis de la demanda y las necesidades de formación; diseñar un proyecto de formación; la definición de los métodos y medios a implementar; coordinación y seguimiento de la formación; la evaluación de la formación así como los modos de validación previstos "(p. 8), queremos proporcionar una herramienta para perfilar a los docentes de FLE en cuanto a la influencia de su cultura disciplinar, su epistemología personal, sus conocimientos pedagógicos y competencias didácticas, y sus conocimientos en el uso de las Tecnologías de la Información y la Comunicación (TIC)

\section{Palabras Clave:}

Ingeniería, formación, marco referencial, docente, competencias

\footnotetext{
${ }^{a}$ Edward Amador Pliego, Universidad Autónoma del Estado de Hidalgo, https://orcid.org/0000-0002-4932-9574, Email: edward_amador@uaeh.edu.mx.

b Delphine Pluvinet, Universidad Autónoma de Querétaro, https://orcid.org/0000-0001-7083-8691, Email:
} pluvinet.delphine@uaq.edu.mx 


\begin{abstract}
:
In recent years, new social and academic situations have emerged in Mexico, allowing a more competitive mobility for language students, particularly French, promoting exchanges and diverse learning objectives.

To face these new situations and considering the lack of formal training in teaching, many teachers are making great efforts to integrate new strategies into their planning and class dynamics, allowing students to better adapt to these recent contexts.

This research shares the first findings initiated at the end of 2019 as part of a Meeting of Bachelor's Degrees in French as a Foreign Language (FFL) in Mexico. As a result of this national meeting, many questions arose about the training and profile of FFL teachers in Mexico, which defined the paper that is being presented here.

The main objective of the scope of the research is to analyse the needs, design actions and training schemes, as well as their implementation and evaluation, based on a grounded and structured approach with the participation of the teachers who were involved in our work.

This work started with an attempt to establish the current profile of the FFL teacher in Mexico. The sample to build this profile was made up of 100 professors from eight public universities in various states of the country. The observations of these teachers regarding the "ideal" profile of a FFL teacher in Mexico were considered as well as their own training trajectories as FFL teachers were reviewed. In addition, directors, coordinators, and heads of language schools and centres were interviewed to identify their concepts, definitions, and perceptions about the ideal professional profile teachers should have to teach classes at their institutions in self-learning centres or FFL laboratories.

This research also aims to propose a new representation of the profile of FFL teachers in Mexico which can easily be used to understand the different influences that determine the pedagogical practices of teachers based on various existing models, but mainly from the principles and tools of Training Engineering. Parmentier (2012) states that "One must understand the methods and practices of analysis of demand and training needs; design a training project; the definition of the methods and means to be implemented; coordination and monitoring of training; the evaluation of the training, as well as the expected validation modes" (p. 8). Because of the above-mentioned, the intention is to provide FFL teachers with a tool that enhances their profile integrating their disciplinary culture, personal epistemology, pedagogical knowledge, and teaching skills, as well as their knowledge in the use of Information and Communication Technologies (ICTs).
\end{abstract}

\title{
Keywords:
}

Engineering, training, referential framework, teacher, competences

\section{INTRODUCCIÓN}

Repensar y proponer un nuevo perfil del profesor de francés en México requiere, ante todo, conocer y evaluar el perfil existente. La lengua francesa siempre ha gozado de gran prestigio en México, tanto porque ha permitido un acercamiento a la cultura occidental y universal, como por la ampliación de posibilidades profesionales que ofrece, al ser una de las lenguas de uso más extendido a nivel mundial (Organisation Internationale de la francophonie, 2019). Actualmente, el francés es la segunda lengua extranjera que más se enseña en México (tan sólo por detrás del inglés) y se imparte en varios niveles educativos en instituciones públicas y privadas (Ramírez Romero, 2010).

En los últimos años, tanto el perfil del docente como los métodos de enseñanza de la lengua francesa han dado un giro importante, al tomar como punto de referencia el Marco Común Europeo de Referencia para las Lenguas (MCER) (Cansigno Gutiérrez, 2018). Sin embargo, en este importante proceso de adopción del MCER, el interés adecuado y necesario en la formación docente no ha evolucionado al mismo ritmo que el interés en la enseñanza y aprendizaje del francés como lengua extranjera.
Tomando en cuenta el universo de necesidades diversas de la enseñanza del FLE, así como las diferencias sociales, culturales y económicas de cada región de un país tan grande como México, ciertamente resultaría complicado definir todos los perfiles y formaciones adecuadas (o más difícil aún, un único perfil y una sola formación) para que los docentes de FLE puedan adaptarse a los requerimientos de todos los estudiantes e instituciones a lo largo y ancho del país (Moerman, 2011).

\section{OBJETIVOS DE LA INVESTIGACIÓN}

Los objetivos de nuestra investigación son los siguientes:

1.1 Exponer algunas de las carencias y necesidades de los docentes de francés en México para que, en un futuro cercano, se les pueda guiar hacia los nuevos enfoques para la enseñanza del FLE, mediante una formación adecuada que permita una mejor adaptación al MCER y una mayor calidad de la enseñanza del FLE.

1.2 Proponer un conjunto de iniciativas para plantear un nuevo perfil de los profesores de FLE en México, que sirva para 
comprender las diferentes influencias que determinan las prácticas pedagógicas de los docentes (FIPF, 2015) y guiar el trabajo de los formadores de los actores involucrados en la enseñanza del francés (coordinadores, consejeros pedagógicos, profesorado, docentes en ejercicio y en formación, asistentes de enseñanza de lenguas).

Para ello se requiere involucrar a los actores mismos de la enseñanza del francés, principalmente a los docentes, en esta fase crítica y constructiva de su propio quehacer docente. Sin pretender desviar el enfoque de nuestra investigación, estamos conscientes de que este trabajo es de gran envergadura y no se puede disociar de la política educativa lingüística, que necesariamente está ligada a la voluntad política del país. Sin embargo, en este artículo nos enfocaremos principalmente en el análisis de las necesidades evidenciadas por los docentes (Leconte, 2015).

\section{METODOLOGÍA}

Se requiere de una metodología bien definida para llevar a cabo la recolección de datos y la puesta en marcha del proyecto, por lo que se han decidido utilizar las herramientas que ofrece la Ingeniería de la Formación para hacer el análisis de la demanda y de las necesidades, el diagnóstico, la concepción del proyecto, el diseño de las acciones y la evaluación que se desarrollará (Ardouin, 2017, 2003, Le Boterf, 1999).

Esta metodología en el campo de la formación data de la década de 1985 y todavía requiere difusión y reconocimiento en el campo de la educación (Le Boterf 1990, Viallet 1987). Por tanto, la ingeniería de formación incluye:

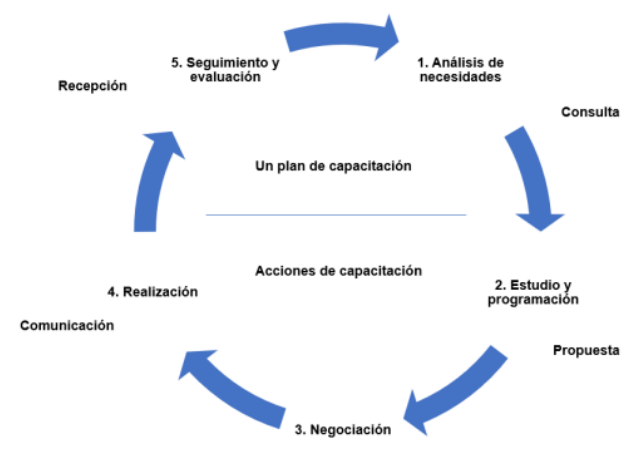

a) Los métodos de análisis de la demanda

En un principio se intentó recabar información a partir de documentos obtenidos directamente de las instituciones (universidades, escuelas privadas, centros de idiomas, escuelas superiores, escuelas secundarias y primarias) referente a la situación de la enseñanza del francés y de la formación de los docentes de FLE. La tarea fue ardua y puso en evidencia una frágil, casi inexistente, comunicación y divulgación de las investigaciones y trabajos realizados al respecto.

Muchos de esos trabajos no tienen una adecuada difusión y, en su mayoría, no llegan a todas las instancias a las que deberían llegar (Silva, 2012; Cansigno Gutiérrez, 2018; Trottet, 2011). De igual forma, se pudo constatar que muchas instituciones educativas no cuentan con un documento que defina el perfil del personal docente de FLE que se requiere; así como tampoco con un programa, una guía o un catálogo de los cursos de formación continua ofertados a sus docentes. Este primer enfoque metodológico nos permitió, sin embargo, establecer comunicación con algunos de los actores de la enseñanza del francés en México, lo que nos facilitó la aplicación de una encuesta entre los docentes de FLE para recopilar información acerca de los propios participantes y poder hacer un análisis posterior. Asimismo, la base de información que obtuvimos nos ha permitido determinar algunas de las necesidades de capacitación más demandadas por los docentes y las instituciones de enseñanza de FLE en México.

El análisis de las necesidades de formación

Ahora bien, para el propósito del trabajo de investigación aquí reportado, se llevó a cabo una etapa de recolección de datos, la cual consistió en la aplicación de una encuesta diseñada en lengua francesa y distribuida mediante la herramienta tecnológica Google Forms, a 100 docentes de francés de diversas instituciones públicas y privadas del país de diferentes niveles educativos.

La finalidad de esta recolección de información era conocer de primera mano la formación que tienen los docentes de FLE en México, así como las carencias que ellos consideraban que tenían en su desarrollo profesional para lograr sentirse totalmente preparados para lograr sus objetivos como docentes. Esto nos permitiría esbozar el perfil actual de los docentes de FLE en México.

Desde el inicio de la recolección de datos se constata que la mayoría de los docentes que respondieron a la encuesta laboran en instituciones públicas, principalmente universidades $(51.5 \%$ de respuestas), mientras que sólo el 7.3\% labora en universidades privadas. Asimismo, el $88.2 \%$ de los docentes de FLE encuestados trabaja con un público adulto, mientras que sólo el $11.8 \%$ lo hace con niños.

A los docentes participantes en esta etapa del estudio se les pidió contestar libremente las siguientes preguntas:

1. ¿En qué Estado de la República Mexicana trabaja? 


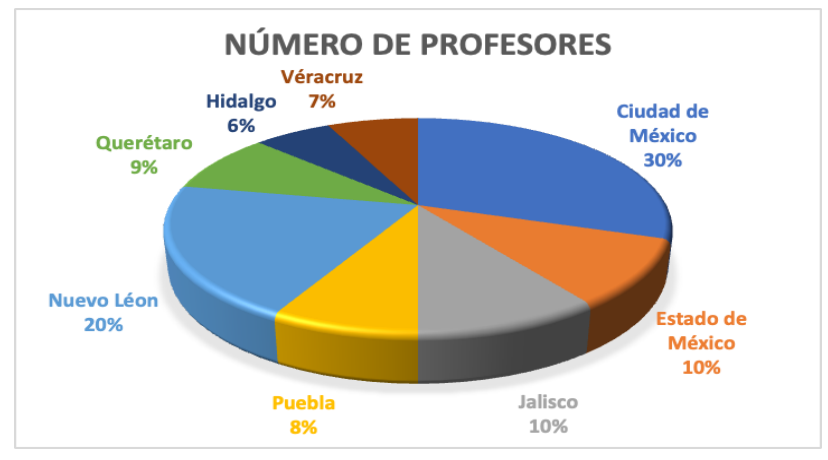

2. ¿En qué sector educativo trabaja?

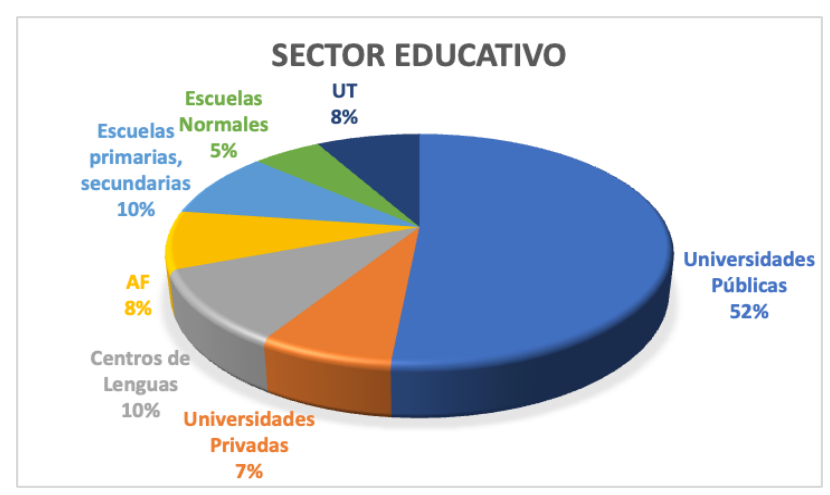

3. ¿A cuántos grupos imparte clase?

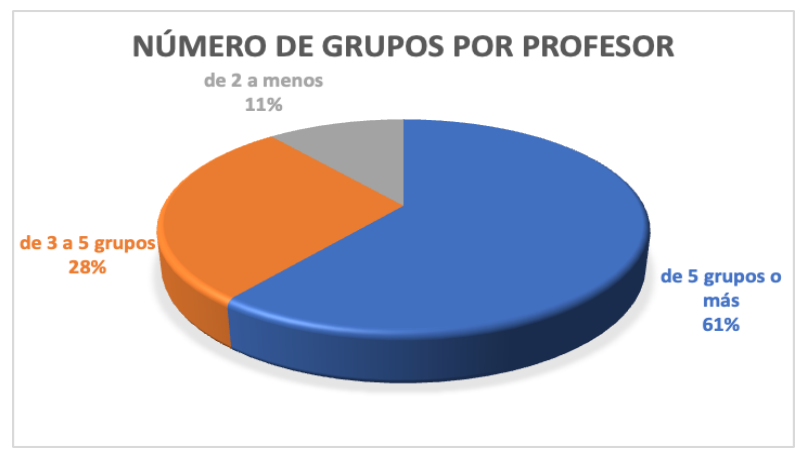

4. ¿Cuántos alumnos tiene en sus grupos?

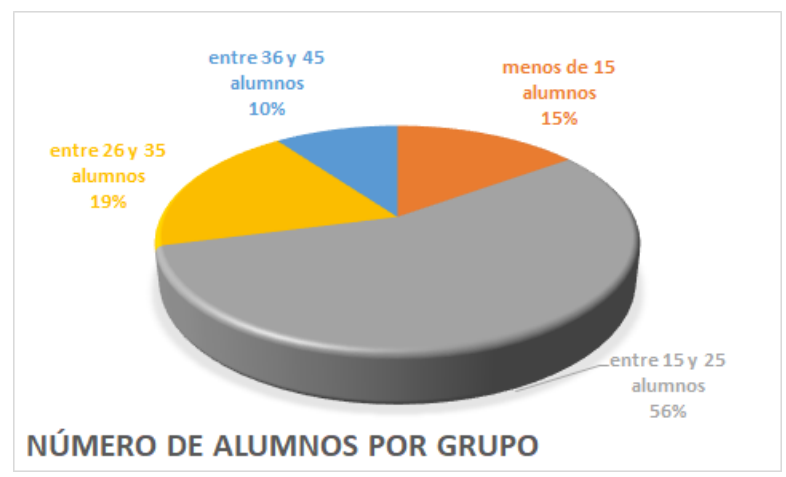

5. ¿Se dedica a otras actividades además de la docencia (coordinadores, examinadores, tutorías, ...)?

\section{ACTIVIDADES EXTRAS ADEMÁS DE LA DOCENCIA}

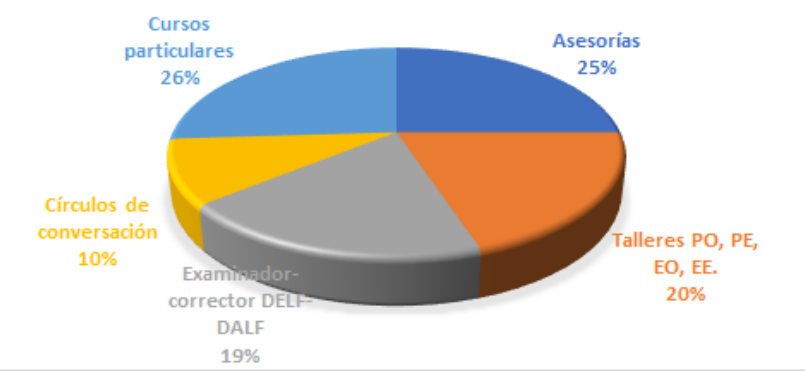

6. ¿Cómo evalúa las formaciones que toman dentro de sus instituciones y fuera de éstas?

\section{PORCENTAJE DE SATISFACCIÓN DE CAPACITACIONES RECIBIDAS}

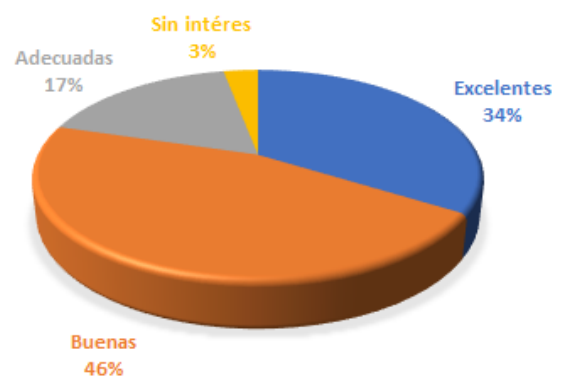

7. ¿Qué le hace falta para dar sus clases?

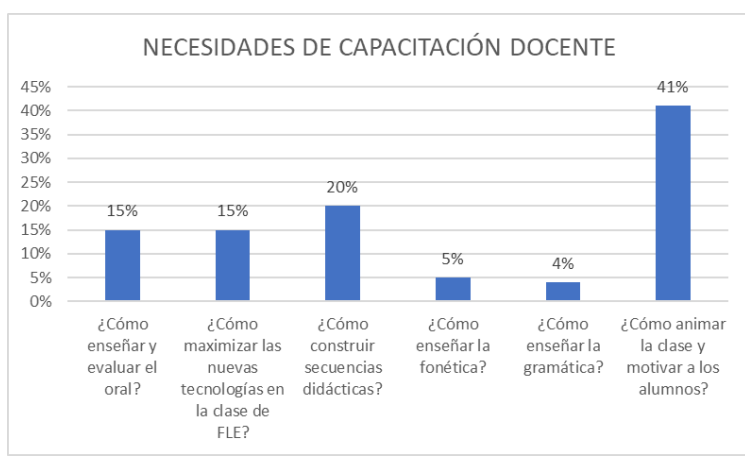


8. ¿Piensa que está bien preparado para las clases que imparte?

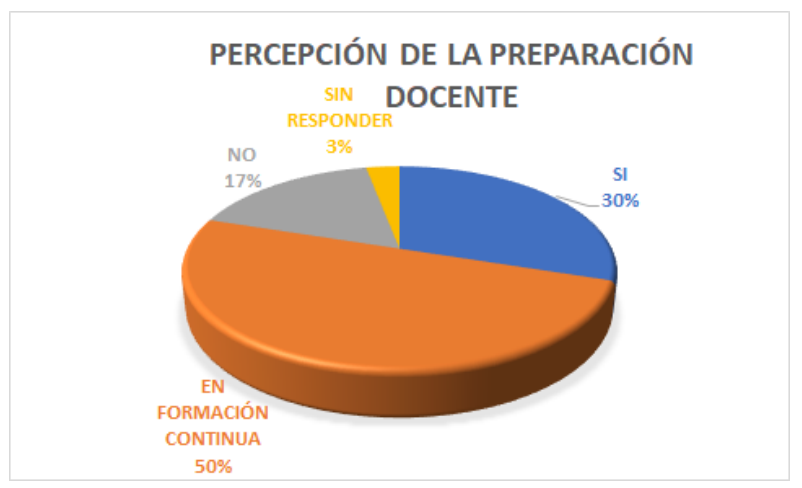

9. ¿Se le exige en sus instituciones un cierto perfil para que se le contrate?

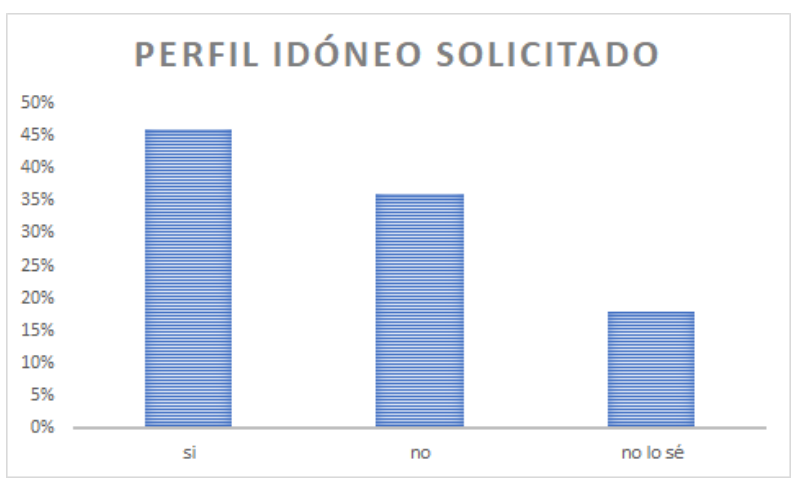

10. ¿Está usted evaluado de acuerdo con la manera de impartir clase y las formas de evaluar a sus estudiantes?

\section{SATISFACCIÓN DE EVALUCIÓN INSTITUCIONAL}

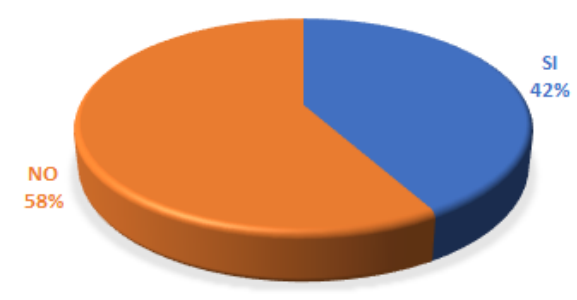

11. ¿Valora las formaciones que les ofrecen sus instituciones?

\section{GRADO DE SATISFACCIÓN DE LAS FORMACIONES OFERTADAS}

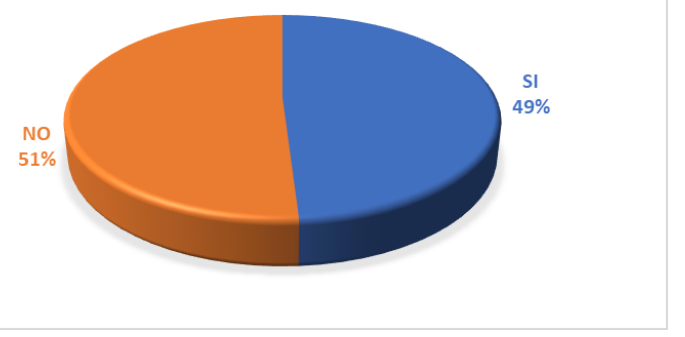

12. ¿Qué herramientas tecnológicas incorpora en sus clases?

\section{HERRAMIENTAS TECNOLOGICAS UTILIZADAS POR LOS DOCENTES}

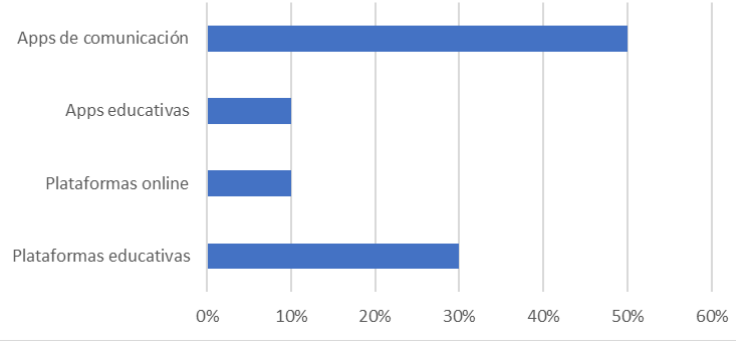

c. El diseño de un proyecto de formación

Una vez obtenidas las respuestas de la encuesta se realizó un primer análisis mediante una metodología cualitativa, buscando recurrencias y puntos comunes entre las respuestas de los participantes. Nuestro análisis se basó en tres ejes diferenciados:

1. Formación de los docentes de FLE;

2. Realidad del contexto laboral de los docentes de FLE y

3. Contraste entre el perfil del docente de FLE y el exigido por la institución.

Tomando en cuenta el eje de formación de los docentes de FLE se analizó la formación de base de los docentes participantes, y si está en relación con el contexto y las clases que imparten. Considerando el eje sobre la realidad del contexto laboral de los docentes, se analizaron las necesidades que tienen los docentes, en qué condiciones realizan su labor y qué tipo de estrategias desarrollan en sus 
entre el perfil del docente de FLE y el exigido por la institución nos ha permitido analizar la coherencia existente entre los perfiles reales de los docentes de FLE y los perfiles deseados por las instituciones donde laboran, así como la importancia que éstas les otorgan para la contratación de sus docentes.

A partir de ese análisis, se hizo un inventario y organizamos las competencias útiles y necesarias, conforme a lo expresado por los docentes participantes, y se empezaron a tratar de definir los rasgos del perfil actual de un docente de FLE en México, así como del perfil idóneo del mismo.

Rápidamente nos dimos cuenta de que teníamos que refinar nuestro grupo de participantes para poder obtener un perfil del actual docente de FLE en México que fuera, a la vez, claro, preciso y conciso. Además, también nos percatamos de que necesitábamos seleccionar una muestra más pequeña de ese primer universo de participantes para poder implementar nuestra primera prueba de formación, conforme a las necesidades expresadas por los participantes.

Así que decidimos afinar el enfoque de nuestro estudio seleccionando a un grupo más reducido de participantes, conformado por 20 docentes de FLE de un centro universitario de enseñanza de lenguas, a quienes se les invitó a participar involucrándose en nuestro estudio, dedicando 5 sesiones de 2 horas cada una para analizar y discutir los resultados de las encuestas realizadas y para, junto con los investigadores, definir las necesidades específicas de formación para la mejora de su labor como docentes de FLE.

d) La definición de los métodos y medios a implementar

Retomando las cuatro etapas sugeridas de la Ingeniería de Formación (Le Boterf, G. 1999):

\begin{tabular}{|l|l|}
\hline \multicolumn{1}{|c|}{ Etapas } & \multicolumn{1}{|c|}{ Actividades } \\
\hline 1. Analizar & $\begin{array}{l}\text { Se analizaron las respuestas sobre la } \\
\text { pertinencia de las formaciones en } \\
\text { relación con las necesidades } \\
\text { enunciadas por los participantes. }\end{array}$ \\
\hline 1. Concebir & $\begin{array}{l}\text { Se definieron los objetivos de la } \\
\text { formación. } \\
\text { Se definieron también los objetivos } \\
\text { pedagógicos. }\end{array}$ \\
\hline
\end{tabular}

\begin{tabular}{|l|l|}
\hline 1. Realizar & $\begin{array}{l}\text { Se elaboró un programa de formación } \\
\text { y el ritmo de las sesiones, en función } \\
\text { de los espacios de tiempo de los } \\
\text { participantes. }\end{array}$ \\
\hline 1. Evaluar & $\begin{array}{l}\text { Se decidió el diseño de la evaluación } \\
\text { y, sobre todo, se pidió a los docentes } \\
\text { ponerlo en práctica con los otros } \\
\text { docentes de FLE de su institución } \\
\text { educativa para definir claramente las } \\
\text { necesidades de capacitación de los } \\
\text { profesores y de las instituciones. }\end{array}$ \\
\hline
\end{tabular}

e) La coordinación y seguimiento de la formación

Los veinte profesores de lengua francesa que participaron voluntariamente en la investigación trabajan en un centro de enseñanza de lenguas adscrito a una universidad pública, por lo que sus cursos están dirigidos a un público adulto de un amplio rango de edades; con diversos intereses y motivaciones por aprender la lengua. Todos los docentes participantes toman constantemente cursos de actualización y reflexionan sobre su propia práctica docente; además, todos ellos muestran una gran implicación y un gran interés en su trabajo, por lo que continuamente analizan nuevas técnicas y estrategias didácticas para tratar de implementarlas en sus clases de lengua.

Debido a la situación sanitaria que se ha vivido en México -y en el Mundo- en los últimos diecisiete meses, hemos tenido que replantear nuestra investigación y adaptar nuestra metodología a la nueva realidad educativa. Así pues, en lugar de tener sesiones presenciales con los docentes participantes en nuestro estudio, hemos tenido que preparar 5 sesiones de trabajo con ellos, de 2 horas cada una, mediante presencia virtual a través de una plataforma de videoconferencias. El objetivo principal de esas sesiones fue analizar, juntamente con ellos, las necesidades de formación para la mejora de su labor como docentes de FLE, de modo que en una segunda etapa podemos proponerles algunos talleres de formación más específica y sesiones de reflexión adhoc a los participantes. En una tercera etapa, con base en la formación recibida y la reflexión sobre su práctica docente, los profesores participantes implementarán nuevas técnicas y estrategias de enseñanza en sus propias clases y nos reportarán sus observaciones sobre el desarrollo de las mismas, lo que nos permitirá hacer un análisis de los resultados obtenidos. 
En las sesiones de trabajo realizadas durante la primera etapa (“Analizar"), algunos de los temas que surgieron recurrentemente son los siguientes:

- ¿Qué tipo de evaluación se debe aplicar para que ésta sea parte del aprendizaje del estudiante?

- ¿Qué herramientas y estrategias didácticas se pueden aplicar para animar grupos-clase grandes?

- ¿Qué herramientas tecnológicas me ofrece la institución donde trabajo para diseñar, organizar y desarrollar mis clases? $¿$ Y si no tengo la formación adecuada necesaria?

- Al no ser francófono nativo, siento que no tengo el conocimiento y las habilidades suficientes para trabajar las competencias de comunicación oral con mis estudiantes.

En la segunda etapa ("Concebir") decidimos proponer los siguientes cuatro talleres de formación:

Taller 1:

¿Qué se debe evaluar en una clase de lengua y cómo hacerlo?

Taller 2:

¿Cómo manejar el grupo-clase, considerando la autonomía de los estudiantes como punto de referencia?

Taller 3:

¿Cómo mi institución puede influir en mi quehacer docente?

Taller 4:

YO, profesor especialista en enseñanza de lengua. (En este taller se valora el conocimiento y dominio de la lengua, así como las competencias docentes, propias de cada profesor participante, y cada uno de ellos comparte a los demás alguna estrategia que emplea con éxito en sus clases de lengua para el desarrollo de las competencias de comunicación oral de sus estudiantes).

f) La evaluación de la formación

A partir de nuestro trabajo hemos detectado una falta de documentación oficial y actualizada de la enseñanza de FLE y del perfil del docente de FLE en México.

Por otro lado, con base en las respuestas de los participantes, se puede constatar una falta de formación especializada y adecuada para los docentes de FLE por parte de las instituciones educativas donde laboran, así como un sentimiento de falta de valoración de la profesión. Asimismo, al parecer de los encuestados no se le da la misma importancia a todas las lenguas extranjeras que se enseñan en las instituciones. De manera general, los participantes señalaron que existen limitaciones personales e institucionales de los docentes de FLE para acceder a una formación superior universitaria en el ámbito de la enseñanza del FLE, y varios de ellos también comentaron que hay una falta de reconocimiento para los profesores que cuentan con un posgrado en la enseñanza de FLE (especialidad, maestría o doctorado) obtenido en el extranjero.

Los participantes también señalaron que hay una oferta limitada de cursos o talleres de formación docente en la enseñanza de FLE, tanto a nivel interno de las instituciones donde laboran como a nivel externo. Asimismo, los docentes manifestaron que existe una cierta incoherencia entre las observaciones y/o evaluaciones institucionales respecto de su labor como profesores de francés y los (pocos) documentos oficiales existentes.

g) Los modos de validación previstos.

\section{CONCLUSIONES}

A partir de los resultados obtenidos hasta ahora, podemos decir que existe un contraste entre los (escasos) esquemas de capacitación actuales y los perfiles de los docentes de FLE expresados en los pocos documentos oficiales existentes en México, con las necesidades expresadas por los propios docentes e instituciones. Nuestro estudio pretende evaluar y adaptar las estructuras ya existentes y dar respuesta a necesidades concretas $\mathrm{y}$ reales.

Esta investigación está apenas en sus inicios ya que la pandemia derivada del COVID-19 nos ha obligado a detener el proceso de formación de los participantes de nuestro estudio; todavía falta para presentar resultados concluyentes. Tiene como principal objetivo evaluar nuestra metodología para proponer una nueva representación del perfil de los docentes de FLE en México a partir de la comprensión de las diferentes influencias que determinan las prácticas pedagógicas de los docentes. Los análisis preliminares de las respuestas y opiniones vertidas por los participantes de este estudio parecen reflejar la necesidad de hacer del enseñante de FLE el observador principal de su propia práctica docente.

Los resultados que se deriven de este estudio se podrán utilizar para diseñar programas de formación para los docentes de FLE adecuados a cada necesidad institucional, pero también para la autoformación de los profesores.

\section{REFERENCIAS}

[1] Ambassade de France au Mexique (2018). Une ambition pour la langue française et le plurilinguisme. Recuperado de https://mx.ambafrance.org/Une-ambition-pour-la-languefrancaise-et-le-plurilinguisme/.

[2] Ardouin, T. (2017). L'ingénierie de formation : Intégrez les nouveaux modes de formation dans votre pédagogie, (ed. 5a), Francia, Dunod. 
[3] Ardouin, T. (2003). L'ingénierie de formation pour l'entreprise : analyser, concevoir, réaliser, évaluer, Francia, Dunod.

[4] Cansigno Gutiérrez, Y. (Coord.) (2018). Le français au Mexique : hier et aujourd'hui. Contribution à un premier état des lieux. México : UAM-Azcapotzalco. 397 pages. ISBN : 978607-28-1356-4.

[5] FIPF (Fédération Internationale des Professeurs de Français (2015). Revue : Dialogues et cultures $n^{\circ} 61$. La formation initiale des enseignants de français langue étrangère. Recuperado de http://fipf.org/sites/fipf.org/files/d_c_ndeg61_-_2015.pdf.

[6] Le Boterf, G. (1990). L'ingénierie de formation et l'évaluation. Les Editions d'Organisation.

[7] Le Boterf, G. (1999). Les défis posés à l'ingénierie de formation et à la production des expertises collectives. Quelles évolutions prendre en compte ? Quelles conséquences pratiques ? Journée d'étude : Ingénierie des dispositifs de formation à l'international. Novembre 1999, Montpellier. Recuperado de http://www.agropolis.fr/formation/pdf/Le_Boterf.pdf.

[8] Leconte, A. (2015). La formation des enseignants de français langue étrangère dans l'éducation de base au Mexique. Le français à l'université 20-03.

[9] Moerman, C. (2011). Les représentations professionnelles des enseignants de FLE de l'Institut français d'Amérique latine : entre idéal professionnel et contraintes institutionnelles, Synergies Mexique núm. 1, GERFLINT, 71-80. Recuperado de https://gerflint.fr/Base/Mexique1/moerman.pdf

[10] Organisation Internationale de la francophonie (2019). La langue française dans le monde. Gallimard/OIF. Recuperado de https://www.francophonie.org/la-langue-francaise-dans-lemonde-2019-en-ligne-en-version-integrale-1067.

[11] Parmentier, C. (2012). L'ingénierie de formation. Collection Ressources humaines. (ed. 2a), Francia. Groupe Eyrolles.

[12] Ramírez Romero, J.L. (coord.) (2010). Las investigaciones sobre la enseñanza de las lenguas extranjeras en México: una segunda mirada. México, CENGAGE Learning / Universidad de Sonora / Universidad Autónoma del Estado de Morelos / Universidad Autónoma del Estado de Hidalgo. 354 pages. ISBN : 978-607-481-262-6.

[13] Silva, H. (2011). Le statut de la langue française au Mexique : esquisse d'une problématique, Synergies Mexique núm. 1, GERFLINT, 17-26. Recuperado de http://ressources-cla.univfcomte.fr/gerflint/Mexique1/haydee_silva.pdf.

[14] Trottet, S. (2011). La professionnalisation des spécialistes de l'enseignement du français au Mexique : vers l'élaboration d'un référentiel de compétences, Synergies Mexique núm. 1, GERFLINT, 35-46. Recuperado de http://ressources-cla.univfcomte.fr/gerflint/Mexique1/soline.pdf.

[15] Viallet, F. (1987). L'ingénierie de la formation. Francia. Les Editions d'Organisation. 